\title{
Valoración de autocuidados en el acceso vascular para hemodiálisis
}

\author{
José Luis García Araque ${ }^{1}$, David Sancho Cantus² \\ ${ }^{1}$ Enfermero, Máster en enfermería, ERESA Grupo Médico, ${ }^{2}$ Doctor en Enfermería. Profesor de la Facultad de \\ Enfermería de la Universidad Católica San Vicente Mártir. Valencia. España
}

\begin{abstract}
Resumen
Introducción: Diversos estudios han evidenciado la falta de formación y en consecuencia la falta de autocuidados en los pacientes renales sometidos a hemodiálisis y con acceso vascular. La enfermería debe asumir su rol de entrenadora de pacientes crónicos para que alcancen una situación de equilibrio entre sus deseos y lo que deben hacer, para que así puedan asumir la responsabilidad en la autoprovisión de los cuidados básicos del mismo. El objetivo fue evaluar los conocimientos que tiene el paciente sobre las necesidades de cuidado de su acceso vascular.
\end{abstract}

Metodología: El presente estudio se realizó en el Centro de Hemodiálisis Fresenius Sagunto situado en la población de Canet D'en Berenguer (Valencia).Se utilizaron dos instrumentos de medición; un cuestionario para pacientes con fístula arteriovenosa, y otro para pacientes con catéter venoso central, donde se incluyen aspectos generales del autocuidado, acciones para mantener el acceso vascular en óptimas condiciones y medidas generales de aplicación en caso de emergencia. Los cuestionaros fueron recogidos durante el periodo comprendido entre el 1 de junio y el 5 de junio del 2015. Se realizó un muestreo por selección intencionada y se consiguió la colaboración de 57 pacientes.

Resultados: El nivel de conocimientos de autocuidados del acceso vascular de los pacientes fue alto en la mayoría de los casos. Los pacientes fueron predominantemente de sexo masculino, pertenecientes a la tercera edad, de estado civil casado, tenían un nivel de estudios básicos, tenían una fístula arteriovenosa como acceso vascular y disponían de bastante experiencia y tiempo de tratamiento.

Correspondencia:

José Luis García Araque

E-mail: josgarar@hotmail.com
Conclusiones: Obtenemos como conclusión final que la hipótesis planteada: "una deficiencia de autocuidados del paciente del centro de hemodiálisis Fresenius Sagunto sobre su AV como consecuencia de un déficit de conocimientos" no se cumple en la muestra de pacientes estudiados.

PALABRAS CLAVE

- HEMODIÁLISIS

- ACCESO VASCULAR

- AUTOCUIDADO

Assessment of self-care in the hemodialysis vascular access

\section{Abstract}

Introduction: Several studies have shown the lack of formation and, in consequence, the lack of selfcare in renal patients in treatment with hemodialysis and with vascular access. Nursing must assume its rol of chronic patients coach in order they achieve a balanced state between their desires and what they must do, so they can assume the responsibility to self -provide their own sef-care. The objective was to evaluate the knowledge that the patient has about the needs of care of his/her vascular access.

Methodology: The current study was implemented in the Fresenius Sagunto Hemodialysis Center located in Canet D'en Berenguer town (Valencia). Two tools were used; a questionnaire for patients with an arteriovenous fistula, and another one for patients with a central venous catheter, in which it was included general aspects of self-care, actions to keep the vascular access in optimal state and general measures of application in case of emergency. The questionnaires were collected during the period 
between the first of June and the fifth of June in 2015. It was made a sampling by intentional selection; the collaboration of 57 patients was achieved.

Results: The level of knowledge of the self-care of the vascular access from the patients was high in most of the cases. The patients were male the most of them, seniors, their civil status was married, they had a elementary level of studies, they had an arteriovenous fistula as a vascular access and they possessed quite experience in treatment time.

Conclusions: We reach to the final conclusion that the raised hypothesis: "a lack of self-care in the patient of Fresenius Sagunto Hemodialysis Center about his vascular access as a consequence of a lack of knowledge" it can't be kept in the sample of patients studied.

\section{PALABRAS CLAVE}

- HEMODIALYSIS

- VASCULAR ACCESS

- SELF-CARE

\section{Introducción}

La enfermedad renal crónica (ERC) o insuficiencia renal crónica (IRC) es un deterioro progresivo e irreversible de la función renal. Cuando la tasa de filtración glomerular es menor de $15 \mathrm{ml} / \mathrm{min}$ se requiere un tratamiento de sustitución de la función renal para el mantenimiento de la vida ${ }^{1}$.

Hay tres principales opciones para el tratamiento sustitutivo renal: hemodiálisis (HD), diálisis peritoneal (DP) y trasplante renal. El trasplante renal es generalmente aceptado como la forma óptima de tratamiento sustitutivo renal para pacientes con enfermedad renal terminal. Sin embargo, dado el rápido aumento de la incidencia y prevalencia de la enfermedad renal terminal y la escasez de riñones de donantes, la mayoría de los pacientes con enfermedad renal terminal necesitarán algún tipo de diálisis: DP o HD².

En la actualidad, el uso de DP como técnica de diálisis en España, es claramente desproporcionada con respecto a la HD. A pesar de ser una técnica realizada por el paciente en su propia casa y que requiere menos recursos, está poco desarrollada en nuestro país ${ }^{3,4}$.
En HD, es necesario un acceso vascular (AV) que permita la conexión del sistema circulatorio del paciente al equipo de HD. Para ello se necesita un alto flujo de sangre, de fácil conexión y punción, duradero, repetible y libre de complicaciones: infecciones, trombosis, estenosis, etc. Se puede utilizar una fístula arteriovenosa (FAV) autóloga: realizada con los propios vasos del paciente mediante anastomosis entre una arteria y una vena o protésica: realizada con un injerto, 0 un catéter venoso central $(\mathrm{CVC})^{5,6}$.

Las complicaciones relacionadas con el AV causan en los pacientes en HD un elevado coste y una alta morbilidad. En los últimos años, a pesar de los avances técnicos en HD, su supervivencia no ha mejorado. La causa es que la edad y la comorbilidad, con el aumento de la presencia de HTA y DM, de estos pacientes cada vez es mayor ${ }^{5,7}$.

Dorothea Elizabeth Orem (1914-2007) creadora de la teoría del autocuidado, abordó la relación entre necesidades y habilidades del paciente para mantener las condiciones para el buen funcionamiento y desarrollo de la vida. Cuando una persona no cumple con las habilidades suficientes para satisfacer una demanda de autocuidado, es necesario que otra persona realice tal atención. Esa derivación no asegura la ausencia de déficit de éste ${ }^{8}$.

Diversos estudios han evidenciado la falta de formación y en consecuencia la falta de autocuidados en los pacientes renales sometidos a HD y con AV. La enfermería debe asumir el rol de entrenadora de pacientes crónicos y también hace de puente entre familiares y tutores de los pacientes fomentando el diálogo fluido, siendo la comunicación familiar un arte de enfermería que también tendrá un impacto significativo en la calidad de cuidados del paciente ${ }^{8,9,10,11}$.

Por tanto, ante la hipótesis: "existe una deficiencia de autocuidados del paciente del centro de hemodiálisis Fresenius Sagunto sobre su AV como consecuencia de un déficit de conocimientos", nuestros objetivos fueron evaluar los conocimientos que tiene el paciente sobre las necesidades de cuidado de su AV.

\section{Material y métodos}

El presente estudio de tipo observacional, descriptivo, transversal, se realizó en el Centro de Hemodiálisis Fresenius Sagunto, situado en la población de Canet D’en Berenguer (Valencia). 
Se utilizaron dos instrumentos de medición; un cuestionario para pacientes con $\mathrm{FAV}$, y otro para pacientes con CVC, en donde se incluyen aspectos generales del autocuidado, acciones para mantener el AV en óptimas condiciones y medidas generales de aplicación en caso de emergencia. Ambos cuestionarios fueron sometidos a una prueba piloto y evaluados para obtener índices de confiabilidad mediante la prueba de Spearman-Brown. Las respuestas fueron cerradas para correcto "sí" o incorrecto "no" y "no sabe". El cuestionario: "Recomendaciones que debe seguir un paciente que tiene una FAV para HD", cuenta con 29 ítems con la siguiente escala de medición: muy alto 25-29 puntos, alto 19-24, medio 1318, bajo $7-12$ y muy bajo 0-6 puntos. El cuestionario: "Recomendaciones que debe seguir un paciente que tiene un CVC para HD", cuenta con 27 ítems, con la siguiente escala de medición: muy alto: 23-27 puntos, alto: 18-22, medio 13-17, bajo: 7-12 y muy bajo 0-6 puntos ${ }^{10}$.

Los cuestionaros fueron recogidos durante el periodo comprendido entre el 1 de junio y el 5 de junio del 2015. Se realizó un muestreo por selección intencionada. Se disponía en el centro de 82 pacientes en ese momento y fueron estudiados todos los posibles, obteniendo una muestra de 57 pacientes.

Los criterios de exclusión fueron: datos no recogidos entre las fechas: 01/06/2015 y 05/06/2015, menores de edad de 18 años, pacientes que reciben tratamiento fuera del centro Fresenius Sagunto, pacientes que no firman el consentimiento informado, pacientes que no pueden realizar su propios autocuidados (dependientes de cuidadores), pacientes con AV tipo FAV protésica, pacientes con deterioro congnitivo que no entienden el proyecto ni las preguntas formuladas.

El estudio se realizó de acuerdo con los Principios Básicos para toda Investigación Médica, Declaración de Helsinki y de acuerdo con la Ley Orgánica de Protección de Datos de Carácter Personal ${ }^{12}$. También se respetaron los derechos y obligaciones en materia de información y documentación sanitaria ${ }^{13}$ y la Ley de Derechos e información al Paciente de la Comunidad Valenciana ${ }^{14}$. Además, a los pacientes voluntarios se les proporcionó la información del proyecto y se les requirió que firmaran el documento de Consentimiento informado. Previo a todo, se consiguió la aprobación por parte del Comité Ético de Investigación Clínica (CEIC) del Hospital de Sagunto (num. registro: EI_2015_04).

\section{Resultados}

La muestra constó de 57 pacientes. De los cuales el $63^{\prime} 16 \%$ fueron hombres. El $56^{\prime} 14 \%$ de estado civil casado frente al $22^{\prime} 81 \%$ viudos, $15^{\prime} 79 \%$ solteros y $5^{\prime} 26 \%$ separados/divorciados. El 84'21\% tenía un nivel de estudios básicos frente al $8^{\prime} 77 \%$ analfabetos y un $7^{\prime} 02 \%$ con estudios universitarios o de formación profesional. El 82'46\% tenían un FAV como AV frente al $17^{\prime} 54 \%$ que tenían un CVC. El 56'14\% de los pacientes obtuvieron un nivel de conocimientos de autocuidados de su AV alto, el $40^{\prime} 35 \%$ un nivel medio, el $3^{\prime} 5 \%$ bajo o muy alto y el $0 \%$ un nivel muy bajo. La edad media fue de 67'98 años. El paciente de más edad fue de 87 años y el de menos de 23 años. La mayoría tenían una edad entre 70 y 87 años. El 25\% de los pacientes tenía más de 79 años. La edad más repetida fue de 75 años. La media de meses en HD fue de $62^{\prime} 51$ meses ( $5^{\prime} 2$ años). La gran mayoría de los pacientes no llevaban más de 10 años en tratamiento. La mayoría llevaban entre 1 y 50 meses.

Para ver si había relación entre las variables: tipo de $A V$, tiempo en HD, nivel de estudios, estado civil y sexo con respecto al nivel de conocimientos de autocuidados se realizó Chi-cuadrado de Pearson y no se vieron diferencias significativas $(p>0.05)$. Al convertir el nivel de conocimientos de autocuidados en variable dicotómica (nivel alto o muy alto: sí o no) sí se vieron diferencias significativas $(p=0.033$ ) y la estimación de riesgo concluyó que los pacientes de sexo femenino tuvieron 3'576 veces más riesgo de tener un nivel de conocimientos de autocuidados del AV "alto o muy alto" que los de sexo masculino. Las mujeres obtuvieron un $76^{\prime} 2 \%$ de conocimientos "altos o muy altos" frente al $47^{\prime} 2 \%$ de los hombres.

Para relacionar la edad y el tiempo en $\mathrm{HD}$ con el nivel de conocimientos de autocuidados primero se realizó el test de normalidad de Shapiro-Wilk y se comprobó que no se cumplían los criterios de normalidad $(p<0.05)$. Así se realizó la prueba de Kruskal Wallys para ambos y con una $p>0.05$ se concluyó que no había relación entre ellas, así que la comparación de medias no fue significativa.

\section{Discusión}

Aunque la media de edad del paciente en HD es cada vez mayor, y en consecuencia su deterioro cognitivo para procurar su autocuidado 5 y que otros estudios constatan por lo general una deficiencia en los auto- 
cuidados de estos pacientes 8,10 , en el presente estudio se determinó que el nivel de conocimientos de autocuidados del AV de los pacientes fue alto en la mayoría de los casos. Aunque cuesta encontrar bibliografía que determine un estudio con similares resultados, en un hospital de Perú se determinó mediante entrevista un nivel de conocimientos altos de autocuidados del $A V^{15}$. Así como en éste, en otros estudios realizados en otros países parece coincidir la predominancia del sexo masculino en los pacientes en $\mathrm{HD}^{15,16}$. El perfil del paciente también fue de un nivel de estudios básicos y de estado casado en su mayoría comparado con otro estudio muy similar realizado en México ${ }^{10}$. La gran mayoría de los pacientes tuvieron una FAV como AV frente al $\mathrm{CVC}^{15}$. Esto se debe a que la FAV nativa se considera el mejor acceso para $\mathrm{HD}^{17}$. También se confirmó con este estudio que la gran mayoría de los pacientes pertenecen a la tercera edad. Siendo una minoría los pacientes de mediana edad ${ }^{15}$. Es habitual que los pacientes en un centro de hemodiálisis lleven de media bastante tiempo en tratamiento. Aunque es difícil encontrar pacientes con más de 10 años ${ }^{15}$.

Una limitación posible del estudio fue que aunque la recogida de datos y la administración de ambos cuestionarios fue realizada por el mismo investigador y siempre en las mismas condiciones no fue realizada por un segundo investigador o colaborador ajeno al centro. Ello podría implicar una posible vinculación personal con los pacientes. Otra limitación fue la imposibilidad de obtener un tamaño muestral mayor al obtenido. Con un número de 82 pacientes en tratamiento en ese momento y aplicando los criterios de inclusión y exclusión, se obtuvieron 57 pacientes voluntarios para la muestra. Aunque el cuestionario es de cuestiones cortas, con respuestas cerradas y fácil de valorar, los pacientes podrían tener cierto reparo en contestar sinceramente y podrían intentar dar la respuesta que ellos intuyeran correcta.

Aun obteniendo un resultado relativamente satisfactorio en el nivel de conocimientos de autocuidados del $A V$, se puede dejar la puerta abierta a una futura línea de investigación más exhaustiva sobre los ítems donde se han encontrado más fallos, dudas o deficiencias. Con ello se podría obtener unas implicaciones prácticas, como ayudar a reforzar el rol de enfermería en la formación de los pacientes y establecer un programa formativo adecuado, elaborando planes educativos de autocuidados, según las deficiencias detectadas. La implementación de modelos de autocuidados aumenta la calidad de vida de los pacientes en hemodiálisis ${ }^{18}$.
La enfermería juega un papel clave en el desarroIlo y mantenimiento del buen funcionamiento y supervivencia del $\mathrm{AV}$ en estos enfermos que cada vez representa un número mayor y de los que la mayoría no llegarán a ser candidatos a un trasplante renal ${ }^{19}$. Una de las funciones más importantes de la enfermería es la educación sanitaria, entre las que debe figurar la enseñanza a los pacientes de los autocuidados del AV. El personal de enfermería que trabaja en las unidades de HD realiza un tratamiento principalmente técnico, pero no debe olvidar la labor educacional. El control de la infección en estos pacientes es un reto para el personal sanitario ${ }^{20}$, ya que es una causa común de muerte entre los pacientes en $H D^{21}$. El enfermero/a es la persona directa de referencia del paciente y en quien deposita una confianza especial. Siendo capaz de gestionar una posible angustia, aportándole la información que necesita de una manera completa, sencilla y clara. Al ser quien pasa más tiempo con el paciente, también es capaz de valorar mejor la capacidad de la persona para comprender la información y las consecuencias. A mejor comprensión por el personal de enfermería de la experiencia del tratamiento del paciente, mejor será la atención dada y mayor la efectividad en la satisfacción de sus necesidades 22, 23, 24 .

\section{Conclusión}

El nivel de conocimientos de autocuidados del AV de los pacientes fue alto en la mayoría de los casos y prácticamente el resto de ellos obtuvo un nivel medio. Sólo una minoría obtuvo un nivel muy alto, bajo o muy bajo.

No hubo relación entre las distintas variables y el nivel de conocimiento de autocuidados del AV, excepto en el sexo del paciente con respecto a tener un nivel de conocimientos altos o muy altos. Así, se vio que la gran mayoría de las mujeres obtuvieron un nivel de conocimientos altos o muy altos frente a los hombres que apenas llegaron a la mitad de los casos en nivel de conocimientos altos o muy altos.

Obtenemos como conclusión final que la hipótesis planteada: "una deficiencia de autocuidados del paciente del centro de hemodiálisis Fresenius Sagunto sobre su AV como consecuencia de un déficit de conocimientos" no se cumple en la muestra de pacientes a estudio. 
Recibido: 2 agosto 2015

Revisado: 20 agosto 2015

Modificado: 22 agosto 2015

Aceptado: 23 agosto 2015

\section{Bibliografía}

1. Tamadon MR, Beladi Mousavi SS. Stability of Renal Function in Spite of Low Glomerular Filtration Rate: A Case Report. Iranian Red Crescent Medical Journal. 2015;17 (2):e21604. doi:10.5812/ ircmj.21604.

2. Beladi Mousavi SS, Hayati F, Valavi E, Rekabi F, Mousavi MB. Comparison of survival in patients with end-stage renal disease receiving hemodialysis versus peritoneal dialysis. Saudi J KidneyDisTranspl. 2015 Mar-Apr;26(2):392-7.

3. Remón-Rodríguez C, et al. Results of the cooperative study of Spanish peritoneal dialysis registries: analysis of 12 years of follow-up. Nefrología. 2014;34(1):18-33. doi: 10.3265/Nefrología. pre2013.Jul.12106.

4. Portolés J, Janeiro $D$, Lou LM, López $P$, Ortega $M$, Del Peso $G$, et al. Primer episodio de infección peritoneal: descripción y factores pronósticos. Nefrología.2013;33:316-24. doi: 10.3265/ Nefrología.pre2013.Feb.11733.

5. Pérez-García R, et al. Estudio epidemiológico de 7316 pacientes en hemodiálisis tratados en las clínicas Fresenius Medical Care de España, con los datos obtenidos mediante la base de datos EuCliD®: resultados de los años 2009-2010. Nefrología 2012;32(6):743-53.doi:10.3265/ Nefrología.pre2012.Jul.1154.

6. Nawaz S, Ali S, Shahzad I, Baloch MU. Arterio venous fistula experience at a tertiary care hospital in Pakistan. Pakistan Journal of Medical Sciences. 2013;29(1):161-165. doi:10.12669/ pjms.291.2753.

7. Santoro D, Benedetto $F$, Mondello $P$, et al. Vascular access for hemodialysis: current perspectives. International Journal of Nephrology and Renovascular Disease. 2014;7:281-294. doi:10.2147/IJNRD.S46643.

8. Costa, Sibely Rabaça Dias da; Castro, Edna Aparecida Barbosa de. Self-care in family caregiver of dependent adults or elderly persons after hospital discharge. Rev Bras Enferm. 2014 Nov-Dec; 67(6):979-86. doi: 10.1590/00347167.2014670617. Epub 2014 Dec 1.

9. Loghmani L, Borhani F, Abbaszadeh A. Factors Affecting the Nurse-Patients' Family Communication in Intensive Care Unit of Kerman: a Qualitative Study. Journal of Caring Sciences. 2014;3(1):67-82. doi:10.5681/jcs.2014.008.

10. Rivera-Ayala L, Lozano-Rangel 0, GonzálezCobos R. Nivel de conocimientos de pacientes con hemodiálisis sobre autocuidado con acceso vascular. Rev Enferm Inst Mex Seguro Soc 2010; 18 (3): 129-135.

11. Ferrer-Arnedo C, Santamaría-García JM, Fernández-Batalla M, Salazar-Guerra R. The value of nursing care in the paradigm of chronicity and dependency. New roles and redesigns. Invest Educ Enferm. 2014 Dec;32(3):488-97. doi: 10.1590/ S0120-53072014000300014.

12. Ley Orgánica $15 / 1999$, de 13 de Diciembre de Protección de Datos de Carácter Personal, publicada el 14 de diciembre de 1999 en el BOE, núm. 298.

13. Ley Orgánica 41/2002, de 14 de noviembre. Ley básica reguladora de la autonomía del paciente y de derechos y obligaciones en materia de información y documentación clínica, publicada el 15 de noviembre de 2002 en el BOE, num. 274.

14. Ley $1 / 2003$, de 28 de enero, de la Generalitat, de Derechos e información al Paciente de la Comunidad Valenciana. (2003/1150). (DOCV núm. 4430 de 31.01.2003).

15. Muro L, Bravo M, Constantino F. Nivel de conocimientos de Autocuidado de las personas en tratamiento de Hemodiálisis. Hospital Almanzor Aguinaga Asenjo. Chiclayo - 2010. Monográficos de investigación en salud. ISSN: 1988-3439-AñoVII-N.19- 2013 Disponible en: http://www.index-f.com/para/n19/292d.php.

16. Arechabala MC, Catoni MI, Palma E, Barrios S. Depression and self-perceived burden of care by hemodialysis patients and their caregivers. RevPanam Salud Pública. 2011 Jul;30 (1):74-9. 
17. Nasir Mahmood S, Naveed Mukhtar K, Igbal N, Umair SF. Pre dialysis care and types of vascular access employed in incident hemodialysis patients: a estudy from Pakistan. Pak J Med Sci. 2013 May;29(3):828-31.

18. Bahadori M, Ghavidel F, Mohammadzadeh S, Ravangard $R$. The effects of an interventional program based on self-care model on health-related quality of life outcomes in hemodialysis patients. J EducHealthPromot. 2014 Nov 29;3:110. doi: 10.4103/2277-9531.145899. e Collection 2014.

19. Beigi AA, Sharifi A, Gheri H, Abdollahi S, Esfahani MA. Placement of long-term hemodialysis catheter (permcath) in patients with end-stage renal disease through external jugular vein. AdvBiomed Res. 2014 Dec 6;3:252. doi: 10.4103/2277-9175.146381. e Collection 2014.

20. Gauna TT, Oshiro E, Luzio YC, Paniago AM, Pontes $E R$, Chang MR. Bloodstream infection in patients with end-stage renal disease in a teaching hospital in central-western Brazil. Rev Soc Bras Med Trop. 2013 Jul-Aug;46 (4):426-32.
21. Fysaraki M, Samonis G, Valachis A, Daphnis $E$, Karageorgopoulos $D E$, Falagas $M E$, et al. Incidence, clinical, microbiological features and outcome of bloodstream infections in patients undergoing hemodialysis. Int J MedSci. 2013 Sep 20;10(12):1632-8. doi: 10.7150/ijms.6710. e Collection 2013.

22. Lin CC, Han CY, Pan IJ. A qualitative approach of psychosocial adaptation process in patients undergoing long-term hemodialysis. Asian Nurs Res (Korean SocNursSci). 2015 Mar;9(1):35-41. doi: 10.1016/j.anr.2014.10.007. Epub 2015 Feb 7.

23. Dos Santos I, De Paula R, Márcia L. Necessidades de orientação de enfermagem para o autocuidado de clientes em terapia de hemodiálise. Rev. Bras. Enferm. Vol. 64 no.2 Brasília Mar./Apr.2011. http:// dx.doi.org/10.1590/S0034-71672011000200018.

24. Farias de Queiroz CM, Dantas J, De Almeida AB, Da Conceição MI, Brandão AL, Venícios De Oliveira $M$. The adaptation problems of patient sunder going hemodialysis:socio-economicandclinicalaspects. Rev. Latino-Am. Enfermagem vol.22 no.6 Ribeirão. Preto Nov./Dec. 2014 EpubDec 2014. 